\title{
Adaptive Radiotherapy in Locally Advanced Head and Neck Cancers: Impact on Target Volume Shrinkage and Organ at Risk and its Clinical Outcome
}

\author{
Sukhveer Tanwar ${ }^{1} \odot$, Shikha Dhal ${ }^{2} \odot$, Sumit Goyal ${ }^{3} \odot$
}

\begin{abstract}
Positional uncertainties due to tumor shrinkage and weight loss, leads to decrease therapeutic ratio due to higher treatment toxicity and decreased local control rates. Adaptive Radiotherapy refers to changing treatment plan during the treatment sessions to account for this decreased therapeutic ratio. Total of 50 patients were treated with image-guided radiotherapy for locally advanced head and neck cancers. Replan was done at 17 th fraction. The cross-comparison of dosimetric analysis of GTV 100 and PTV 95 was statistically significant. Mean volume reduction rate in ipsilateral parotid and contralateral parotid was in our study mean shrinkage in volume of right parotid 13-10.4 cc, left parotid 14-10.5 cc, respectively. Patients who had TVRR $\geq 20 \%$ at interim replan done after 17 fractions at 4 th week had complete response at 6 months after treatment while in patients with TVRR $<20 \%, 42 \%$ had complete response at 6 months. Out of 50 patients enrolled in the study 32 patients (64\%) had complete response on clinical evaluation and follow-up CT scan.

Keywords: Adaptive, Cancer, Tumor volume.

Journal of Mahatma Gandhi University of Medical Sciences \& Technology (2021): 10.5005/jp-journals-10057-0185
\end{abstract}

\section{INTRODUCTION}

The treatment of unrespectable Head and Neck Cancer (HNC) consists of chemo-radiotherapy (CRT) ${ }^{1,2}$

Radiotherapy (RT) has evolved from conventional to conformal techniques. Conventional radiotherapy techniques have historically used blocks based on bony landmarks and sometimes under dosing. ${ }^{3}$ The target and higher doses to normal tissue with high incidence of adverse reactions. To reduce toxicity and improve therapeutic ratio is better target delineation and sparing of normal tissue. This can be achieved by conformal RT technique like three-dimensional conformal radiotherapy (3DCRT) and intensitymodulated radiotherapy (IMRT) using off-line and online computed tomography (CT) and rescanning to identify visible tumor and organ at Risk. ${ }^{4-8}$

One of the unique aspects of head and neck radiation therapy is that noticeable changes in the anatomy occur during the treatment course. ${ }^{9}$ This is mostly result of the response to the radiation and often combined chemotherapy and positioning uncertainties and weight loss. ${ }^{10}$ Positional changes include shrinkage of the tumor and normal tissue volumes as parotid gland (PG) and other structures. ${ }^{11,12}$

Therefore, there is speculation that the plan created on the initial planning CT may no longer be optimal for changing anatomy during the treatment, and the actual radiation dose delivered to the patient may be significantly different from that in the plan. ${ }^{13,14}$

Adaptive Radiotherapy (ART) refers to changing the radiation treatment plan delivered to a patient during a treatment session to account for change in anatomy like tumor shrinkage, weight loss, change in clinical/palpable tumor volume, ill-fitting mask, or prolonged treatment breaks. Decreased appetite and reduced oral intake contribute to weight loss in cancer patients. Tumor volume

\footnotetext{
${ }^{1-3}$ Department of Radiation Oncology, Mahatma Gandhi Medical College \& Hospital, Jaipur, Rajasthan, India
}

Corresponding Author: Shikha Dhal, Department of Radiation Oncology, Mahatma Gandhi Medical College \& Hospital, Jaipur, Rajasthan, India, Phone: +91 08141685814, e-mail: shikha_dhal08@yahoo.co.in

How to cite this article: Tanwar S, Dhal S, Goyal S. Adaptive Radiotherapy in Locally Advanced Head and Neck Cancers: Impact on Target Volume Shrinkage and Organ at Risk and its Clinical Outcome. J Mahatma Gandhi Univ Med Sci Tech 2021;6(3):79-81.

Source of support: Nil

Conflict of interest: None

may shrink after chemoradiation. The distance between OAR (organ at risk) and PTV (Planning target Volume) decrease. These lead to change in dose distribution to normal surrounding tissues (organ at risk) and toxicities.

\section{Materials and Methods}

Patient history and physical examination were done thoroughly for clinical evaluation and performance status will be measured by Karnofsky Performance scale. ${ }^{15}$

All selected patient were underwent basic set of investigationshematological investigations, X-ray chest, contrast-enhanced computed tomography neck.

All biopsy proven cases stating squamous cell carcinoma were included in the study.

Total 50 patients were treated with image-guided radiotherapy with concurrent chemotherapy (CCRT) [total dose 70Gy/35\# (in 7 weeks)] for locally advanced head and neck carcinomas [T3$\mathrm{T} 4 \mathrm{a}, \mathrm{N} 0-2]$

(c) The Author(s). 2021 Open Access This article is distributed under the terms of the Creative Commons Attribution 4.0 International License (https://creativecommons. org/licenses/by-nc/4.0/), which permits unrestricted use, distribution, and non-commercial reproduction in any medium, provided you give appropriate credit to the original author(s) and the source, provide a link to the Creative Commons license, and indicate if changes were made. The Creative Commons Public Domain Dedication waiver (http://creativecommons.org/publicdomain/zero/1.0/) applies to the data made available in this article, unless otherwise stated. 
Planning CT (CT simulation) scans were acquired pretreatment and interfractional (Replanning) [at 17\# completion].

In 1st session treatment was delivered on the basis of pretreatment $\mathrm{CT}$ planning after generating optimal plan (P1).

In 2nd session [at completion of 17\#] another CT Simulation was done and replanning done on the basis of new images (P2).

Comparing both plan, target volume shrinkage and dose distribution to target volume and normal tissues (OAR) was recalculated.

Outcome was assessed by clinical examination at every month till 6 months and radiologically by CT scan neck at 3 and 6 months.

\section{Dosimetric Analysis}

The dosimetric effects were quantified by comparing dose-volume histograms of $\mathrm{P} 1$ and $\mathrm{P} 2$. The target coverage in these plans was clinically assessed using GTV (Gross tumor volume) V100 and PTV (Planning target volume) V95 (volume receiving $100 \%$ and $95 \%$ of the prescription dose, respectively). ${ }^{16,17}$

\section{Tumor Volume Reduction Rate (TVRR)}

Total gross tumor volume (GTVt) changes between the original and rescan CT were analyzed. Pre-RT GTVt and rescan GTVt values were used to calculate TVRR, which defined as: [pre-RT GTVt-rescan GTVt]/pre-RT GTVt ${ }^{18,19}$

Volume Reduction in OARs:

Pre-RT Volume (V1) - rescan Volume (V2)/ pre-RT Volume (V1)

\section{Result and Discussion}

Intensity-modulated radiation therapy (IMRT)/Image-guided radiation therapy (IGRT) is treatment modality technique of choice for management of head and neck cancers. As we know radiation has biological and structural effect on tumor and normal tissue in patients; further patients has weight loss during treatment. And treatment would be delivered on the plan made on the basis of pre-treatment CT scan only, then normal tissue would get higher radiation dose than expected which can result in unexpected high-grade toxicities. So, replan after 17 fractions can evaluate tumor volume reduction rate (TVRR) which has prognostic significance and reduce dose to normal tissue which leads to decrease in late toxicities.

We observed that the mean pair-wise distribution of GTV 100 Plan 1 vs PTV 95 Plan 1 was $-0.524 c c$ and GTV 100 Replans vs PTV 95 Replan was $-0.446 \mathrm{cc}$. The cross-comparison of dosimetric analysis of GTV 100 and PTV 95 was statistically significant ( $p$ value 0.001 ).

As effect of radiation, tumor shrinks, in this study change in volume of GTV range from $0.90-136.50 \mathrm{cc}$. The mean p-GTV was $28.71 \mathrm{cc}$ with standard deviation (SD) $25.18 \mathrm{cc}$. The replan-GTV volume of $r$-GTV was ranging from $0.80-119.30 \mathrm{cc}$; mean $24.79 \mathrm{cc}$ with SD $22.05 \mathrm{cc}$. The decrease in GTV was ranging from $135.60 \mathrm{cc}$ and $118.50 \mathrm{cc}$, respectively. The decrease in GTV was not statistically significant $(p$ value $=0.409$ ).

Hyebin Lee et al. had documented the patients who achieved locoregional control had a higher TVRR than those with loco-regional failure $(p=0.010)$, and those with the tumor VRR $>35 \%$ achieved significantly higher loco-regional control at 3 years ( $94.4 \%$ vs $72.4 \% ; p=0.018$ ). In our study, we observed that the mean pair-wise distribution of GTV 100 Plan 1 vs PTV 95 Plan 1 was $-0.524 c c$ and GTV 100 Replans PTV 95 Replan was $-0.446 c c$. Patients who had TVRR $\geq 20 \%$ at interim replan done after 17 fractions at 4th week had complete response at 6 months after treatment while in patients with TVRR $<20 \%, 42 \%$ has complete response at 6 months.

Kataria et al. has investigated efficacy and toxicities of adaptive radiotherapy and documented median reductions in gross primary disease volumes on midtreatment scans was 34\%; 16 patients experienced grade three acute mucositis; four patients developed local recurrences, all within the RT field. In our study reduction in GTV was 0.90-136.50cc; 20 patients had grade three acute mucositis; 10 patient had local recurrence and eight had distant metastasis in lung during follow-up.

Castadot et al. also quantified volumetric and positional changes of gross tumor volumes (GTV), clinical target volumes (CTV), and organs at risk (OAR) and documented decrease in GTV at a mean rate of $3.2 \%$ and $3.9 \% /$ treatment day $(\mathrm{td})$, respectively; nodal GTVs decreased at a mean rate of $2.2 \% / \mathrm{td}$. The ipsilateral and contralateral parotid glands showed a mean decrease of $0.9 \%$ and $1.0 \% / \mathrm{td}$, respectively. In our study, mean shrinkage in volume of right parotid- 13-10.4cc, left parotid- 14-10.5cc.

Ryota Bando et al. observed a marked reduction of GTV by $28 \%$ of the initial volume was seen by the third week during RT ( $p$-value 0.01).

Shih-Neng Yang et al. has shown primary tumor volume and T staging as a prognostic factors for primary tumor relapse-free survival for OPC (Oropharyngial Carcinoma): T4 tumor ( $p=0.0001$, hazard ratio 7.38), $\mathrm{pGTV} \geq 20 \mathrm{~mL}(p=0.01$, hazard ratio 10.61$) .{ }^{13} \mathrm{In}$ our study, stage III has $85.7 \%$ complete response and $14.3 \%$ partial response whereas stage IV has $36.3 \%$ complete response and $63.7 \%$ has partial response. But we didn't find significant corelation between pretreatment GTV and clinical response.

Joel Castelli et al. showed that the main goal of definitive chemo-radiotherapy in locally advanced HNC (Head and Neck Cancers) is to improve locoregional control, while keeping a high quality of life by reducing the dose in the parotid gland during the whole course of IMRT and therefore xerostomia is a major challenge. Indeed, they found the majority of the parotid gland $(59 \%)$ was being over irradiated by a mean dose of $4 \mathrm{~Gy}$ (up to $10 \mathrm{~Gy}$ ), resulting to an absolute increase risk of xerostomia of $8 \%$ (up to $24 \%$ ). The Adaptive Radiotherapy strategy appears to benefit not only to the overirradiated parotid gland patients, reducing the mean dose of 5 Gy (up to $12 \mathrm{~Gy}$ ) and the xerostomia risk of $11 \%$ (up to $30 \%$ ), but also to the non-over-irradiated parotid gland. These results suggest thus a large use of adaptive radiotherapy for the majority of locally advanced HNC patients.

Qiuwen $\mathrm{Wu}$, et al. stated that multiple factors affect the parotid glands doses. If no replanning is done, then the actual dose ( $D$ mean) delivered to the patient will be $10 \%$ higher than those shown in the initial plan. Reducing margins from 5-0 mm, improve the sparing of the parotid gland by $22 \%$. Therefore, the main benefit of replanning is to preserve the sparing of the parotid show in the initial plan.

Murat Surucu, et al. furthermore correlated TVRR with clinical outcomes and were able to show statistically significant differences in DFS and OS for patients with TVRR $35.2 \%$ and TVRR $>35.2 \%$ control. Thus, by identifying patients with low TVRR with the use of ART, physicians can potentially consider change in therapeutic strategy for this group of patients. This study has identified numerous benefits to ART for patients with HNC, including improvement in tumor coverage, decrease in dose to OARs, and measurement of tumor volume regression rate to potentially tailor therapy for individual patients. 


\section{Conclusion}

The cross-comparison of dosimetric analysis of GTV 100 and PTV 95 was statistically significant.

The decrease in GTV was ranging from $135.60 \mathrm{cc}$ and $118.50 \mathrm{cc}$, respectively. The decrease in GTV was not statistically significant.

Volumetric change was seen in OARs except mandible and spinal cord. All organ at risk have decrease in volume during replan except mandible and spinal cord which was statistically significant.

As we have done only one replan therefore it will be inappropriate to conclude ideal time period for adaptive replan. But this study has concluded that interim replan at 4 weeks (after 17 fractions) appear to be beneficial. Out of 50 patients enrolled in study 32 patients (64\%) had complete response on clinical evaluation and follow-up CT scan after 6 months.

\section{OrCID}

Sukhveer Tanwar $\odot$ https://orcid.org/0000-0003-1554-2649

Shikha Dhal ๑ https://orcid.org/0000-0001-8549-107X

Sumit Goyal ๑ https://orcid.org/0000-0003-1566-5796

\section{References}

1. Bray F, Ferlay J, Soerjomataram I, et al. Global cancer statistics 2018 : GLOBOCAN estimates of incidence and mortality worldwide for 36 cancers in 185 countries. CA Cancer J Clin 2018;68(6):394-424. DOI: 10.3322/caac.21492

2. Spitz MR. Epidemiology and risk factors for head and neck cancer. Semin Oncol 1994;21(3):281-288.

3. Pfister DG, Foote RL, Gilbert J, et al. NCCN Guidelines Index Table of Contents Discussion. 2018;227.

4. Wu Q, Manning M, Schmidt-Ullrich R, et al. The potential for sparing of parotids and escalation of biologically effective dose with intensitymodulated radiation treatments of head and neck cancers: a treatment design study. Int J Radiat Oncol Biol Phys 2000;46(1):195-205. DOI: 10.1016/s0360-3016(99)00304-1

5. Chao KC, Majhail N, Huang C, et al. Intensity-modulated radiation therapy reduces late salivary toxicity without compromising tumor control in patients with oropharyngeal carcinoma: a comparison with conventional techniques. Radiother Oncol 2001;61(3):275-280. DOI: 10.1016/s0167-8140(01)00449-2

6. Dogan N, King S, Emami B, et al. Assessment of different IMRT boost delivery methods on target coverage and normal-tissue sparing. Int J Radiat Oncol Biol Phys 2003;57(5):1480-1491. DOI: 10.1016/s03603016(03)01569-4

7. Li Y, Taylor JM, Ten Haken RK, et al. The impact of dose on parotid salivary recovery in head and neck cancer patients treated with radiation therapy. Int J Radiat Oncol Biol Phys 2007;67(3):660-669. DOI: 10.1016/j.ijrobp.2006.09.021
8. Pow EH, Kwong DL, McMillan AS, et al. Xerostomia and quality of life after intensity-modulated radiotherapy vs. conventional radiotherapy for early-stage nasopharyngeal carcinoma: initial report on a randomized controlled clinical trial. Int J Radiat Oncol Biol Phys 2006;66(4):981-991. DOI: 10.1016/j. ijrobp.2006.06.013

9. Mendenhall WM, Mancuso AA, Morris CG, et al. Influence of tumour volume on the probability of local control after radiotherapy for squamous cell carcinoma of the head and neck. JHK Coll Radiol 2003;6:119-125.

10. Strongin A, Yovino S, Taylor R, et al. Primary tumor volume is an important predictor of clinical outcomes among patients with locally advanced squamous cell cancer of the head and neck treated with definitive chemoradiotherapy. Int J Radiat Oncol Biol Phys 2012;82(5):1823-1830. DOI: 10.1016/j.jjrobp.2010.10.053

11. Lok BH, Setton J, Caria N, et al. Intensity-modulated radiation therapy in oropharyngeal carcinoma: effect of tumor volume on clinical outcomes. Int J Radiat Oncol Biol Phys 2012;82(5):1851-1857. DOI: 10.1016/j.ijrobp.2011.03.029

12. Been MJ, Watkins J, Manz RM, et al. Tumor volume as a prognostic factor in oropharyngeal squamous cell carcinoma treated with primary radiotherapy. Laryngoscope 2008;118(8):1377-1382. DOI: 10.1097/MLG.0b013e318172c82c

13. Yang S-N, Liao C-Y, Chen S-W, et al. Int J Radiat Oncol Biol PhysClinical implications of the tumor volume reduction rate in head-andneck cancer during definitive intensity-modulated radiotherapy for organ preservation. 2011;79(4):1096-1103. DOI: 10.1016/j. ijrobp.2009.12.055

14. Lee $H, A h n Y C, O h D$, et al. Tumor volume reduction rate measured during adaptive definitive radiation therapy as a potential prognosticator of locoregional control in patients with oropharyngeal cancer. Head Neck 2014;36(4):499-504. DOI: 10.1002/ hed.23328

15. Péus D, Newcomb N, Hofer S. Appraisal of the Karnofsky performance status and proposal of a simple algorithmic system for its evaluation. BMC Med Inform Decis Mak 2013;13(1):72. Available from: http://bmcmedinformdecismak.biomedcentral.com/ articles/10.1186/1472-6947-13-72 DOI: 10.1186/1472-6947-13-72

16. ICRU-50. ICRU Report 50. 1993;

17. Cox JD, Stetz J, Pajak TF. Toxicity criteria of the radiation therapy oncology group (RTOG) and the European organization for research and treatment of cancer (EORTC). Int J Radiat Oncol Biol Phys1995;31(5):1341-1346. DOI: 10.1016/0360-3016(95)00060-C

18. O'Daniel JC, Garden AS, Schwartz DL, et al. Parotid gland dose in intensity-modulated radiotherapy forhead and neck cancer: is whatyou plan what you get? Int J Radiat Oncol Biol Phys 2007;69(4):1290-1296. DOI: 10.1016/j.ijrobp.2007.07.2345

19. Dijkema T, Raaijmakers CP, Ten Haken RK et al. Parotid gland function after radiotherapy: the combined Michigan and Utrecht experience. Int J Radiat Oncol Biol Phys 2010;78(2):449-453. DOI: 10.1016/j. ijrobp.2009.07.1708 